\title{
Development of a Fluorescent Tool for Studying Legionella bozemanae Intracellular Infection
}

\author{
Breanne M. Head ${ }^{1}$ (D), Christopher I. Graham ${ }^{2}$, Teassa MacMartin ${ }^{2}$, Yoav Keynan ${ }^{1, *}$ and Ann Karen C. Brassinga ${ }^{2}$ \\ 1 Department of Medical Microbiology and Infectious Diseases, University of Manitoba, Winnipeg, MB R3T \\ 2N2, Canada; Umheadb@myumanitoba.ca \\ 2 Department of Microbiology, University of Manitoba, Winnipeg, MB R3T 2N2, Canada; \\ Graham13@myumanitoba.ca (C.I.G.); Macmartt@myumanitoba.ca (T.M.); \\ Ann.brassinga@umanitoba.ca (A.K.C.B.) \\ * Correspondence: Yoav.keynan@umanitoba.ca
}

Citation: Head, B.M.; Graham, C.I.; MacMartin, T.; Keynan, Y.; Brassinga, A.K.C. Development of a Fluorescent Tool for Studying Legionella bozemanae Intracellular Infection. Microorganisms 2021, 9, 379. https://doi.org/ 10.3390 /microorganisms 9020379

Academic Editors: Janet E. Stout and Julianne L. Baron

Received: 31 December 2020

Accepted: 11 February 2021

Published: 13 February 2021

Publisher's Note: MDPI stays neutral with regard to jurisdictional claims in published maps and institutional affiliations.

Copyright: (c) 2021 by the authors. Licensee MDPI, Basel, Switzerland. This article is an open access article distributed under the terms and conditions of the Creative Commons Attribution (CC BY) license (https:/ / creativecommons.org/licenses/by/ $4.0 /)$.
Abstract: Legionnaires' disease incidence is on the rise, with the majority of cases attributed to the intracellular pathogen, Legionella pneumophila. Nominally a parasite of protozoa, L. pneumophila can also infect alveolar macrophages when bacteria-laden aerosols enter the lungs of immunocompromised individuals. L. pneumophila pathogenesis has been well characterized; however, little is known about the $>25$ different Legionella spp. that can cause disease in humans. Here, we report for the first time a study demonstrating the intracellular infection of an L. bozemanae clinical isolate using approaches previously established for L. pneumophila investigations. Specifically, we report on the modification and use of a green fluorescent protein (GFP)-expressing plasmid as a tool to monitor the L. bozemanae presence in the Acanthamoeba castellanii protozoan infection model. As comparative controls, L. pneumophila strains were also transformed with the GFP-expressing plasmid. In vitro and in vivo growth kinetics of the Legionella parental and GFP-expressing strains were conducted followed by confocal microscopy. Results suggest that the metabolic burden imposed by GFP expression did not impact cell viability, as growth kinetics were similar between the GFP-expressing Legionella spp. and their parental strains. This study demonstrates that the use of a GFP-expressing plasmid can serve as a viable approach for investigating Legionella non-pneumophila spp. in real time.

Keywords: Acanthamoeba castellanii; GFP; intracellular infection; Legionella; Legionella bozemanae; non-pneumophila

\section{Introduction}

Legionella are Gram-negative, rod-shaped bacteria that cause Legionnaires' disease (LD), which is a severe type of pneumonia that remains a global and public health concern. In recent years, cases of Legionnaires' disease have increased at an alarming rate, with outbreaks having been reported across North America, Europe, and Australia [1-3]. Although the exact reason for this increase is unknown, it is likely multifactorial, representing increased disease awareness and diagnostic testing, in addition to an increase in the susceptibility of the global population, as well as global warming, and aging infrastructure, both of which favor Legionella growth in the environment. In Canada, Legionella incidence has risen 10-fold since 2000, with 635 cases having been reported to the Public Health Agency of Canada in 2018 [4]. Incidence has also increased 9-fold in the United States (US) and 2-fold in Europe [5,6]. Legionella cases are thought to be under-reported for reasons discussed later. A study by Collier et al. [7] estimated that Legionella species (spp.) caused 10,800 (95\% confidence interval (CI) 7280-13,100) patient hospitalizations in the US in 2014, equating to $\$ 401$ million (95\% CI \$79-1690) in total direct healthcare costs, highlighting the significant burden that these organisms have on the healthcare system.

In the environment, Legionella spp. can be found in naturally occurring and man-made water systems such as showers, cooling towers, fountains, lakes, and streams [8]. To survive 
these low-nutrient habitats, they have adapted to parasitize a variety of protozoan species including Vermamoeba, Naegleria, and Acanthamoeba. Once inside their protozoan host, Legionella can subvert the host cellular machinery by creating a replicative niche, known as a Legionella-containing vacuole (LCV), where they can proliferate to high titers until the cell ruptures, facilitating their release back into the environment [9-11].

The Legionella genus is comprised of more than 50 different species, with half of them able to cause disease in humans [12]. However, most available information about Legionella spp. pathogenesis and interaction with its host stems from studies focused on Legionella pneumophila serogroup (SG) 1, which is the type strain [13]. Diagnostics, although previously reliant on culture, have shifted toward the use of the urinary antigen test (UAT). The UAT is a rapid and inexpensive method that has high sensitivity for L. pneumophila SG1, favoring the detection of this organism while leading to false-negative results when patients are infected with other L. pneumophila SG or Legionella spp. [14]. Indeed, studies from North America and Europe, regions which tend to favor the use of the UAT for diagnosing Legionella spp. infections, report L. pneumophila SG1 as the primary cause of LD (>90\% of cases) [15]. This may be more reflective of the diagnostic methodologies rather than a true representation of species diversity. In a study conducted by $\mathrm{Ng}$ et al. [16], which analyzed 1401 cases of Legionella infection between 1978 and 2006, it was found that when culture was used, only $66 \%$ of cases were, in fact, L. pneumophila SG1. The authors suggested that proximity to the Great Lakes played a role in disease epidemiology, which further supports the findings that Legionella epidemiology can vary by region. Indeed, in places such as Australia and New Zealand, other Legionella spp. such as L. longbeacheae are more prevalent in the environment and have been shown to account for up to half of all of the Legionella cases in Australia [2,15]. Consequently, alternative methods such as serology or polymerase chain reaction (PCR) are more commonly used for diagnosing Legionella infections in some of these regions [17].

Infections due to Legionella non-pneumophila spp., such as those caused by L. bozemanae, have most frequently been reported among individuals that have underlying immune suppression, which is associated with malignancy or HIV infection [18], as well as recipients of immunomodulatory treatments $[19,20]$. Aside from causing pneumonia, $L$. bozemanae infection can lead to cavitary lung lesions [21], soft-tissue infections [22], and septic arthritis [21]. However, diagnosing L. bozemanae infections is difficult, since the UAT and serological kits currently do not target L. bozemanae, and traditional culture techniques often result in non-detection due to the use of cefamandole-containing media, which can inhibit the growth of non-pneumophila spp. [21,23]. Consequently, infections due to $L$. bozemanae have primarily been reported as case studies, and information on L. bozemanae virulence and pathogenesis is currently lacking.

Since infection models are well established for L. pneumophila SG1 pathogenesis, we sought to use these models to glean insight into Legionella non-pneumophila infections. As fluorescent-based detection systems have been used extensively to investigate host-cell interactions, bacterial pathogenesis, and protein function in L. pneumophila studies and in other bacterial species [24-27], we aimed to exploit their use for studying L. bozemanae. The green fluorescent protein (GFP) reporter system is a well-documented tool that can be used for fluorescence, since it is bright enough to detect above autofluorescence levels and stable enough to allow measurements over time $[28,29]$. However, it is important to note that optimal use of a GFP-expressing plasmid is best achieved if the strain of interest is verified to be devoid of other plasmids. Legionella species have been reported to have naturally occurring plasmids that may be incompatible with the IncQ-group derived GFP plasmid once introduced [30]. Moreover, although different bacterial species appear to tolerate GFP better than others, the overexpression of GFP in some bacteria can cause toxicity [31].

Here, we describe the development of a GFP reporter plasmid that was transformed into two Legionella clinical isolates (L. bozemanae and L. pneumophila) and the type strain, L. pneumophila American Type Culture Collection (ATCC) 33152. This study serves as a tool 
to create fluorescent Legionella strains, which can be used to characterize non-pneumophila Legionella bacterial growth kinetics in vitro (broth) and in vivo (Acanthamoeba castellanii infection model).

\section{Materials and Methods}

\subsection{Bacteria, Plasmids, Primers, and Growth Conditions}

Bacterial strains and plasmids used in this study are listed in Table 1. Primers and amplicon product sizes are listed in Table 2. Escherichia coli DH5 $\alpha$ strains were grown in Luria-Bertani broth or on agar, supplemented with kanamycin $(40 \mu \mathrm{g} / \mathrm{mL})$ or ampicillin $(100 \mu \mathrm{g} / \mathrm{mL})$ where appropriate, and incubated overnight at $37^{\circ} \mathrm{C}$. Legionella spp. were streaked from frozen stocks (Buffered Yeast Extract (BYE) $+10 \%$ dimethyl sulfoxide) onto Buffered Charcoal Yeast Extract (BCYE) agar at $37^{\circ} \mathrm{C}$ with $5 \% \mathrm{CO}_{2}$ for $3-4$ days then if required, heavily inoculated into BYE broth [32] for $18-24 \mathrm{~h}$ at $37^{\circ} \mathrm{C}$ on a tube rotator, supplemented with kanamycin $(25 \mu \mathrm{g} / \mathrm{mL})$.

Table 1. Organisms and plasmids used in this study.

\begin{tabular}{|c|c|c|}
\hline Organisms & Genotype & Reference/Source \\
\hline $\begin{array}{c}\text { Legionella pneumophila ATCC } \\
\text { 33,152 Philadelphia-1 }\end{array}$ & Parental & ATCC \\
\hline $\begin{array}{c}\text { Legionella pneumophila } 950 \\
\text { clinical isolate }\end{array}$ & Parental & $\begin{array}{c}\text { Health Sciences Centre } \\
\text { (Winnipeg, Canada) }\end{array}$ \\
\hline $\begin{array}{l}\text { Legionella bozemanae } 948 \\
\text { clinical isolate }\end{array}$ & Parental & $\begin{array}{l}\text { Health Sciences Centre } \\
\text { (Winnipeg, Canada) }\end{array}$ \\
\hline LP 1030 & $\begin{array}{c}\text { L. pneumophila ATCC 33,152 } \\
\text { pGFP-Kan; Kan }{ }^{\mathrm{r}}\end{array}$ & This study \\
\hline LP 1031 & $\begin{array}{l}\text { L. pneumophila } 950 \text { pGFP-Kan; } \\
\operatorname{Kan}^{\mathrm{r}}\end{array}$ & This study \\
\hline LB 1032 & $\begin{array}{l}\text { L. bozemanae } 948 \text { pGFP-Kan; } \\
\operatorname{Kan}^{\mathrm{r}}\end{array}$ & This study \\
\hline E. coli $\mathrm{DH} 5 \alpha$ & $\begin{array}{l}\mathrm{F}^{\prime} \text { endA1 hsdR17 }\left(r_{k^{-}} m_{k^{-}}\right) \\
\text {supE44 thi-1 recA1 gyrA (Nalr) } \\
\text { relA1 } \Delta(\text { lacZYA-argF)U169 } \\
\text { deoR( } \Phi 801 \text { lac } \Delta(\text { lacZ)M15) }\end{array}$ & $\begin{array}{c}\text { Laboratory stock } \\
\text { (New England Biolabs) }\end{array}$ \\
\hline $\begin{array}{l}\text { Acanthamoeba castellanii } \\
\text { (ATCC 30234) }\end{array}$ & Douglas & ATCC \\
\hline \multicolumn{3}{|c|}{ Plasmids } \\
\hline p34S-Kan & $\begin{array}{l}\text { Cloning vector with } \mathrm{Kan}^{\mathrm{r}} \\
\text { cassette }\end{array}$ & $\begin{array}{c}\text { Laboratory stock } \\
\text { Dennis et al., } 1998 \text { [33] }\end{array}$ \\
\hline pKB127 & $\begin{array}{l}245 \mathrm{bp} \mathrm{P} \text { magA region cloned } \\
\text { into BamHI and XbaI sites of } \\
\text { pBH6119; } \mathrm{Amp}^{\mathrm{r}}, \mathrm{Thy}^{+}\end{array}$ & Morash et al., 2009 [26] \\
\hline pGFP-Kan & pBH6119 PmagA; Kan ${ }^{\mathrm{r}}$ & This study \\
\hline
\end{tabular}

Note: ATCC, American Type Culture Collection; Bp, base pair; GFP, green fluorescent protein; Kan $^{\mathrm{r}}$, kanamycin resistance; Thy $^{+}$, thymidine auxotroph. 
Table 2. List of primers and expected size of polymerase chain reaction products used in this study.

\begin{tabular}{|c|c|c|c|}
\hline Primer & Sequence $\left(5^{\prime}\right.$ to $\left.3^{\prime}\right)$ & Annealing Temperature $\left({ }^{\circ} \mathrm{C}\right)$ & Amplicon Size (bp) \\
\hline pF KanR EcoRI & CGCATAgaattcCCACGTTGTGTCTCAAAATCTCTG & 60 & \multirow{2}{*}{1120} \\
\hline pR KanR SacII & CGCATAccgcggGGTTGATGAGAGCTTTGTTGTAG & 60 & \\
\hline pR KanR Conf Int & GTGAGAATGGCAAAAGCTTATG & 60 & 179 \\
\hline pF KanR Conf Ext & GTGCCCATTAACATCACCATC & 60 & $1200^{\mathrm{a}}$ \\
\hline pR KanR Conf Rev & GTTATTTCTCCGGATTTAATTCG & 60 & $726^{b}$ \\
\hline
\end{tabular}

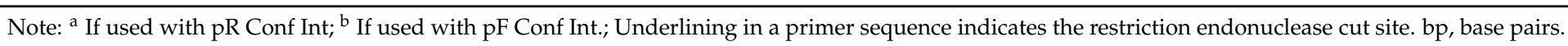




\subsection{Construction of GFP-Expressing Legionella Strains}

In general, L. pneumophila can maintain RSF1010-based plasmids [34,35]. In this view, pKB127 was genetically modified to replace the $t d \Delta I$ marker with a kanamycin resistance marker, sourced from p34S, creating pGFP-Kan for use with the Legionella strains in this study. pKB127 refers to the insertion of a 245 base pair (bp) magA promoter region in the promoterless $g f p$ reporter plasmid pBH6119 [26] and was chosen, since $m a g A$ is a strong promoter that tends to be more expressed post-exponentially. pBH6119 carries a thymidylate synthase $(t d \Delta I)$ that rescues the thymidine auxotrophy of Lp02, which is a derivative of L. pneumophila Philadelphia-1 [36], thereby providing a convenient non-antibiotic-based plasmid selection. However, since Legionella spp. are generally not thymidine auxotrophs, a kanamycin resistance marker was used as a substitute.

Clinical isolates of L. bozemanae and L. pneumophila, along with L. pneumophila ATCC 33,152 type strain, were confirmed to lack intrinsic resistance to kanamycin by plating on BCYE supplemented with kanamycin and checked for growth daily for $96 \mathrm{~h}$.

To generate GFP-expressing Legionella strains, the $\mathrm{P}_{\operatorname{magA}} g f p$ reporter plasmid (pKB127) was genetically modified by replacing the $t d \Delta I$ selection marker with a kanamycin resistance marker. Briefly, pKB127 was cut with EcoRI and SacII to drop out the $t d \Delta I$ selection marker, with the PCR-amplified kanamycin resistance marker ligated in its place, creating pGFP-Kan. E. coli DH5 $\alpha$ was used as the host strain for cloning approaches. Amplification of the kanamycin resistance marker with engineered restriction sites was achieved with primer set PF KanR EcoRI/PR KanR SacII and template p34S using Q5 High Fidelity Polymerase (New England Biolabs, Canada). The construct of pGFP-Kan was verified by polymerase chain reaction (PCR) using a combination of the internal and external primer sets listed in Table 2. Then, the plasmid was electroporated into the L. bozemanae, L. pneumophila clinical, and L. pneumophila ATCC 33,152 type strains according to the methods described in Tanner et al. [35] to create the strains LP 1031, LB 1032, and LP 1030, respectively, which were then plated on BCYE supplemented with kanamycin to select for the kanamycin resistance phenotype. Plasmid integration was confirmed by screening the kanamycin-resistant GFP-expressing Legionella strains with the PCR confirmation primers (Table 2).

\subsection{In Vitro Growth Kinetics and Relative Fluorescence Assays}

In vitro growth of the Legionella parental and GFP-expressing strains was assessed in BYE broth as described previously [32,37]. Briefly, each strain was grown on the appropriate BCYE medium for $96 \mathrm{~h}$ and then resuspended in BYE, either with or without kanamycin, and inoculated into a 96-well plate at a final concentration of $1.5 \times 10^{7}$ cells $/ \mathrm{mL}$ [35] The plate was incubated for $24 \mathrm{~h}$ at $37^{\circ} \mathrm{C}$ with shaking in a BioTek Synergy HTX multimode automated microplate reader and optical density $\left(600 \mathrm{~nm} ; \mathrm{OD}_{600}\right)$ and GFP relative fluorescence (excitation and emission wavelengths $485 / 20 \mathrm{~nm}$ and 528/20 nm, respectively) was measured every hour. Media background fluorescence and absorbance was subtracted from the relative fluorescence unit (RFU) values and optical density $\left(\mathrm{OD}_{600}\right)$ data, respectively, and values for each of the GFP strains along with their parental strains were plotted over time.

\subsection{Intracellular Growth Assays in Acanthamoeba castellanii}

For maintenance of A. castellanii ATCC 30234, amoeba were cultured in ATCC 712 medium, Peptone Yeast Extract Glucose (PYG) liquid medium, at $25^{\circ} \mathrm{C}$ [26]. Amoeba were split every $48-96 \mathrm{~h}$ (depending on the initial seeding dilution). For infections, a 48-h culture of A. castellanii was washed and resuspended in A. castellanii buffer (AC buffer; $4 \mathrm{mM}$ magnesium sulfate, $0.4 \mathrm{mM}$ calcium chloride, $3.4 \mathrm{mM}$ sodium citrate dihydrate, $0.05 \mathrm{mM}$ ferrous ammonium sulfate hexahydrate, $2.5 \mathrm{mM}$ disodium phosphate, and 2.5 $\mathrm{mM}$ potassium dihydrogen phosphate) and then seeded into 24-well plates at a density of $1 \times 10^{6}$ cells $/ \mathrm{mL}$ [10]. Once cells were adherent $\left(\approx 2 \mathrm{~h}\right.$ at $\left.25^{\circ} \mathrm{C}\right)$, bacteria (from 3-4day-old BCYE-agar plates) were suspended in AC buffer, and titers were determined 
at an $\mathrm{OD}_{600}$. Then, amoeba were infected with Legionella parental and GFP-expressing strains at a multiplicity of infection (MOI) of 0.1 . Following a $1 \mathrm{~h}$ incubation at $25^{\circ} \mathrm{C}$, to synchronize the infection, amoeba were washed 3 times in AC Buffer to remove any extracellular bacteria that had not been taken up into the cells [35]. At various time points $(1,48,144$, and $216 \mathrm{~h}$ post-infection), amoeba were lysed using mechanical disruption (i.e., by scraping the monolayer, vortexing the sample for $30 \mathrm{~s}$ followed by centrifugation for $8 \mathrm{~min}$ at 14,800 rpm) [38], and bacterial counts were determined by serial dilution BCYE plate counts (colony-forming units (CFU)/mL).

\subsection{In Vitro and In Vivo Microscopic Imaging of the GFP-Expressing Legionella Strains}

All GFP-expressing Legionella strains were cultured on BYCE agar supplemented with kanamycin for $96 \mathrm{~h}$ prior to in vitro and in vivo imaging studies. For in vitro imaging, a single isolated colony was resuspended in $100 \mu \mathrm{L}$ of BYE and mounted on a glass microscope slide. Then, bacteria were heat-fixed (i.e., by placing a drop of bacteria on a slide, letting it dry, and then passing the slide through a flame 2 times) and mounted in ImmunoMount medium (Thermo Scientific, Waltham, MA, USA) before viewing.

To visualize GFP-expressing Legionella within A. castellanii, the day prior to infection, amoeba were placed in a 6-well plate containing sterile poly-lysine coated coverslips (Neuvitro Corp, Vancouver, WA, USA) at a density of $3 \times 10^{6}$ cells/well and allowed to adhere overnight in a $25{ }^{\circ} \mathrm{C}$ incubator. Then, $A$. castellanii was infected with each GFPexpressing strain at an MOI of 5, and the infection was incubated for 6 or $12 \mathrm{~h}$. Following infection, the cells were washed 3 times with AC buffer, stained with an ER-Tracker Red dye (BODIPY TR Glibenclamide; ThermoFisher Scientific, Whitby, ON, Canada) for $30 \mathrm{~min}$ at $37^{\circ} \mathrm{C}$, and fixed in formaldehyde. Then, the coverslips containing the infected amoeba were mounted onto glass slides with ProLong Gold Antifade Mountant with DAPI (ThermoFisher Scientific, Canada) per the manufacturer's instructions.

For fluorescence imaging, all slides were examined by differential interference contrast (DIC) and confocal microscopy using an LSM 700 confocal microscope with accompanying Zeiss software. GFP-expressing Legionella, the ER-Tracker Red Dye, and DAPI had an excitation and emission spectra of 488/583,587/615, and 405/461 nm, respectively. Images were generated using a $40 \times$ oil immersion objective. All fluorescent images were edited using ImageJ version 1.52A [39].

\subsection{Statistical Analyses}

All experiments were performed in triplicate unless otherwise stated. Data were analyzed using STATA®version 14. Bacterial optical densities and growth (CFU/mL) are reported as the mean and standard error. To determine if optical densities were similar between the fluorescent strains and their parental counterparts at inoculation, a T-test was performed, while a one-way analysis of variance was used to compare optical densities between the Legionella parental strains (L. bozemanae (948), L. pneumophila clinical (950) and L. pneumophila ATCC 33152) as well as those from the GFP-expressing strains (L. bozemanae (LB 1032), L. pneumophila clinical (LP 1031) and L. pneumophila ATCC 33,152 (LP 1030)). To compare bacterial optical densities and fluorescence intensity over time between the GFP-Legionella strains, a repeated-measures analysis of variance was carried out with a Bonferroni post-hoc analysis conducted when data were significant $(p \leq 0.05)$. All data were plotted using GraphPad Prism version 9 (GraphPad Software, San Diego, CA, USA).

\section{Results}

\subsection{Generation of GFP-Expressing Legionella Strains}

Transformation of the Legionella strains by electroporation was carried out with relative ease, requiring one attempt when constructing the GFP-L. pneumophila strains and two attempts for the GFP-L. bozemanae strain. Following electroporation and plating on BCYE supplemented with kanamycin, transformants were streaked out on fresh agar plates for purity, after which frozen stocks were prepared. Fluorescence microscopy confirmed GFP 
expression (Figure 1). Colony characteristics of the GFP isolates, such as colony shape and size, did not appear to differ from their parental strains.

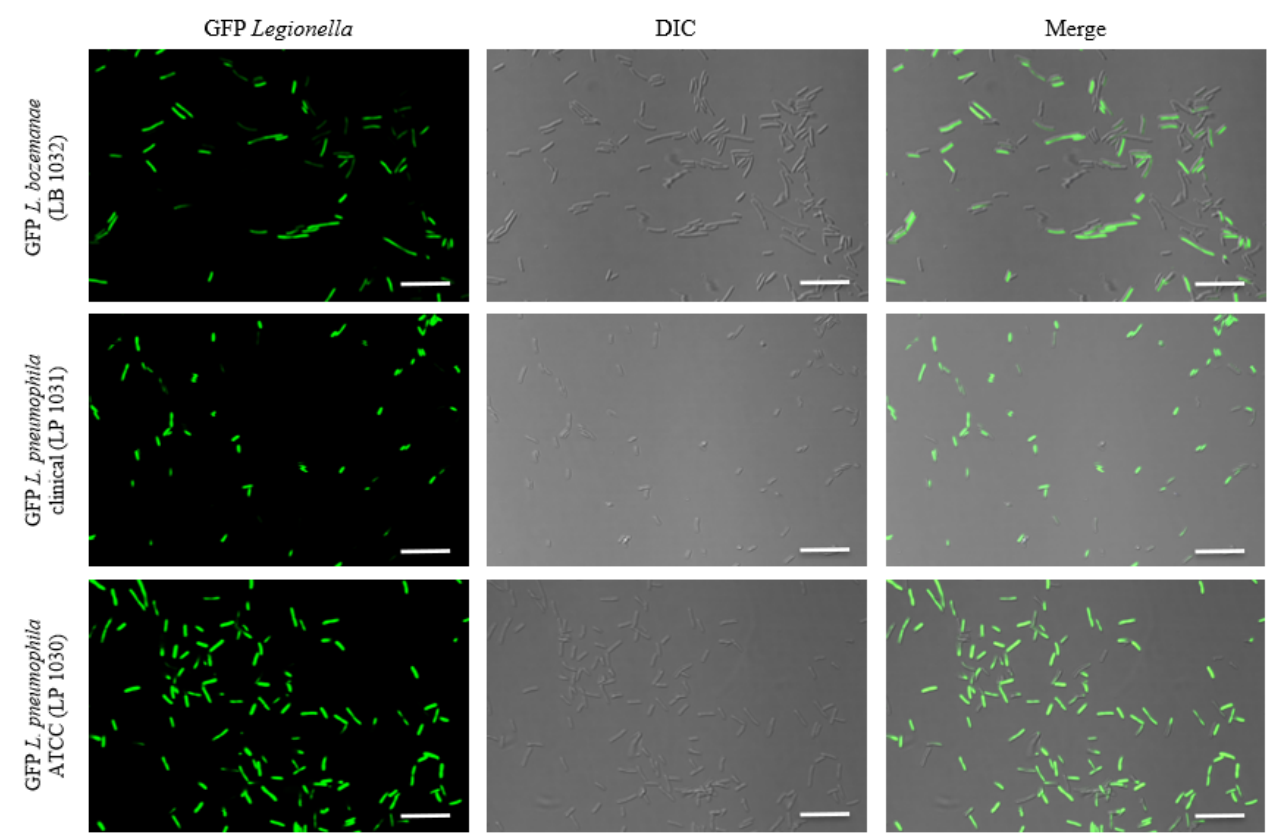

Figure 1. Differential interference contrast (DIC) and confocal microscopy of the GFP-expressing L. pneumophila and L. bozemanae strains imaged with a Zeiss LSM 700 confocal microscope. Images were generated using a $40 \times$ oil immersion objective. The panel on the far right represents the merged DIC and fluorescence channels. The scale bar represents $10 \mu \mathrm{m}$. ATCC, American Type Culture Collection; GFP, green fluorescent protein.

\subsection{Plasmid-Borne GFP Expression Does Not Affect the Growth of Legionella In Vitro}

To determine if the presence of pGFP-Kan affects Legionella growth profiles, bacterial kinetic studies were performed to compare the growth rates of each of the GFP-expressing Legionella strains with their parental counterparts. Each of the Legionella pairs (with and without pGFP-Kan) displayed similar growth curves over the $24 \mathrm{~h}$ period with all of the strains reaching their peak density between 12 and $16 \mathrm{~h}$ (Figure 2A-C). No significant differences were seen between the optical densities of the parental strains and those of their GFP-expressing counterparts.

When comparing the growth profiles of the L. pneumophila parental and transformant strains with that of the L. bozemanae strains, higher densities were seen among the L. pneumophila species compared to those seen with L. bozemanae $\mathrm{OD}_{600}$ of 1.0 vs. 0.5 , respectively, $p<0.0001)$. Likewise, the GFP-expressing L. pneumophila strains also displayed higher rates of fluorescence compared to those of the GFP-expressing L. bozemanae $(p<0.0001)$, with 24-h RFU values of 3385, 3137, and 857 for strains LP 1030, LP 1031, and LB 1032, respectively (Figure 2D-F). None of the Legionella parental strains were fluorescent as evidenced by the RFU values, which mimicked that of the BYE media control. 

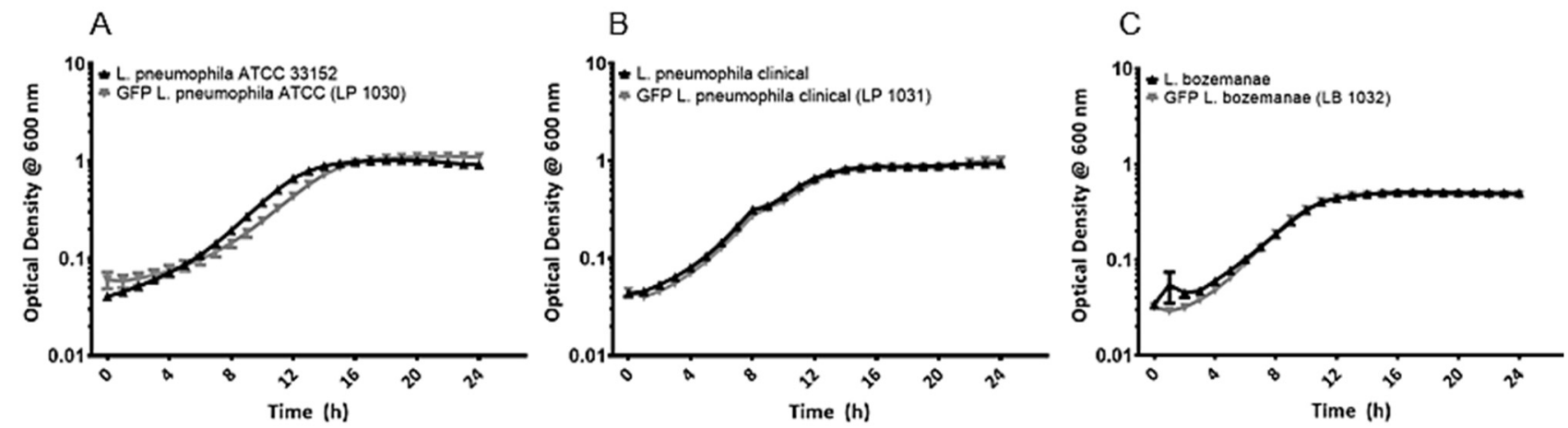

D

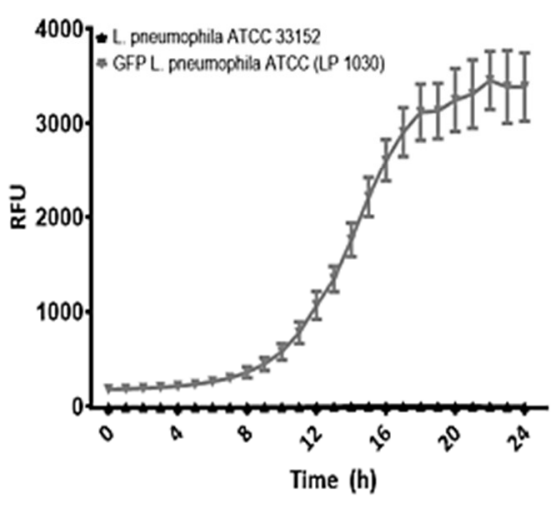

E

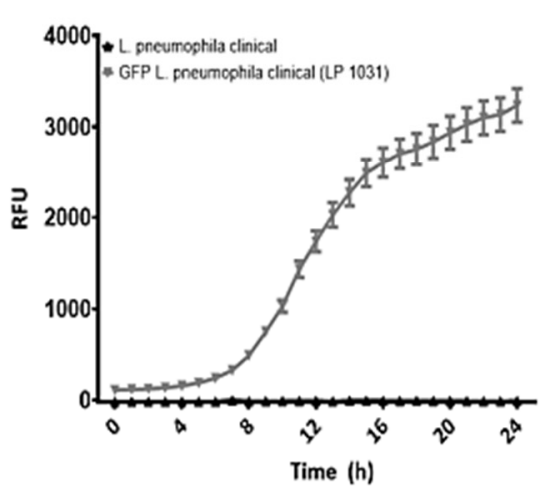

G

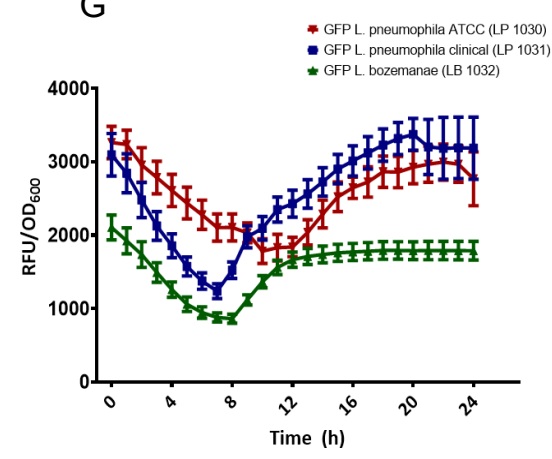

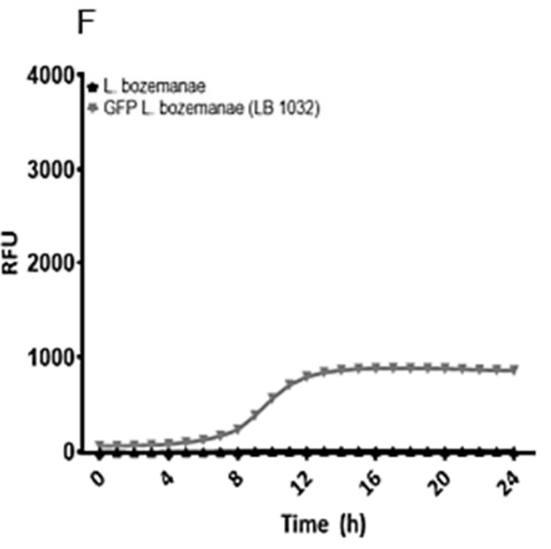

Figure 2. In vitro growth curves and relative fluorescence for the GFP-tagged Legionella and parental strains. All Legionella isolates were incubated in BYE broth with shaking in an automated microplate reader for $24 \mathrm{~h}$ where $\mathrm{OD}_{600}(\mathrm{~A}-\mathrm{C}), \mathrm{RFU}$ (D-F), and RFU/OD $600(G)$ were measured every hour. For panels (A-F), the parental strains are displayed in black, while the GFP-expressing strains are shown in gray. For panel G, the GFP-expressing L. pneumophila ATCC and L. pneumophila clinical isolate are shown in red and blue, respectively, while the GFP-expressing L. bozemanae is in green. All experiments were performed in triplicate and data represent the mean \pm standard errors; ATCC, American Type Culture Collection; BYE, Buffered Yeast Extract; GFP, green fluorescent protein; OD 600, optical density at $600 \mathrm{~nm}$; RFU, relative fluorescent units.

Similarly, when RFU values were normalized to optical density, L. bozemanae fluorescence was consistently lower than that of the GFP-expressing L. pneumophila strains (Figure 2G). Indeed, the initial GFP activity of the LB 1032 strain began at approximately $2000 \mathrm{RFU} / \mathrm{OD}_{600}$ units, while activity was $>3000 \mathrm{RFU} / \mathrm{OD}_{600}$ units for the LP 1031 and LP 1030 strains. Each of the GFP-expressing strains displayed a similar trend over the 24-h experiment: the RFU/OD 600 values decreased throughout the first 7-10 $\mathrm{h}$ of the assay, at which time the RFU/OD 600 values rebounded, increasing gradually until they returned to their approximate starting values. The growth-dependent GFP expression profiles exhibited by all three strains reflect the activity of the mag $A$ promoter, which tends to be higher post-exponentially as reported elsewhere [26]. 


\subsection{Plasmid-Borne GFP Expression Does Not Affect Legionella Growth within A. castellanii protozoa}

To determine if plasmid-borne GFP expression altered Legionella growth and viability in vivo, A. castellanii protozoa were infected with each of the Legionella strains, and intracellular growth kinetics were assessed. Legionella growth profiles appeared to be unaffected by GFP expression. Both parental and GFP-expressing L. pneumophila strains (ATCC 33,152 and clinical) showed similar growth profiles, achieving $10^{7} \mathrm{CFU} / \mathrm{mL}$ by the $216-\mathrm{h}$ time point (Figure 3). Likewise, the GFP-expressing L. bozemanae strain also exhibited a similar growth profile in vivo compared to its parental counterpart.
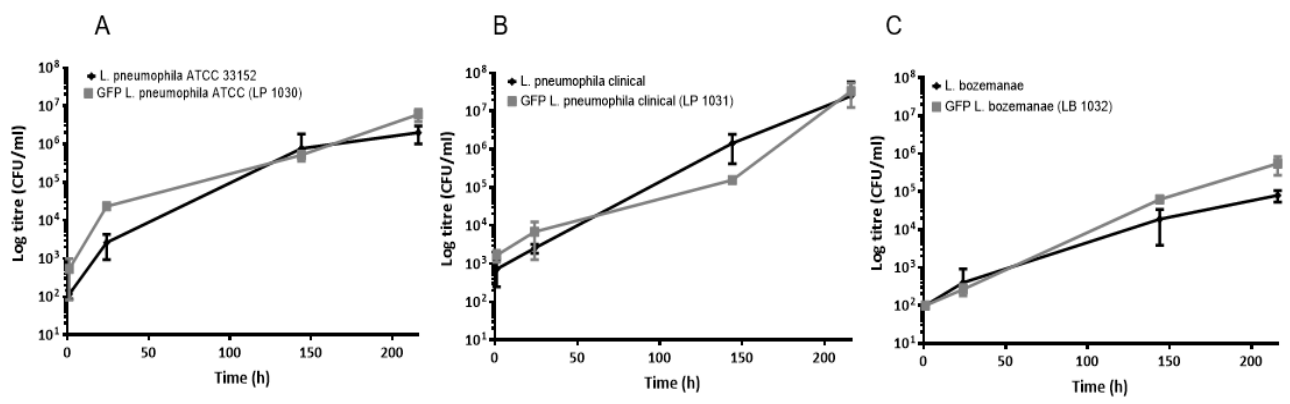

Figure 3. In vivo growth curves for the GFP-tagged Legionella and wild-type strains. Parental strains and GFP-tagged L. pneumophila ATCC 33152 (A), L. pneumophila clinical isolate (B) and L. bozemanae (C) strainswere grown in Acanthamoeba castellanii at $25^{\circ} \mathrm{C}$. Infections were performed at an MOI of 0.1 and Legionella intracellular growth was determined at 1, 48, 144, and $216 \mathrm{~h}$ post-infection. The parental strains are displayed in black, while the GFP strains are shown in gray. All experiments were performed in triplicate, and data represent the mean \pm standard errors. ATCC, American Type Culture Collection; CFU, colony-forming units; GFP, green fluorescent protein; MOI, multiplicity of infection.

However, as was seen with the in vitro experiments, L. bozemanae growth was less efficient than L. pneumophila, which was highlighted by the lower log titers seen with the $L$. bozemanae strains $(p<0.001)$. At each time point, L. bozemanae growth was approximately 1 $\log$ lower than the L. pneumophila species, with L. bozemanae achieving $10^{5}$ and $10^{6} \mathrm{CFU} / \mathrm{mL}$ by the end of the 216-h experiment for the parental and fluorescent strain, respectively. These results indicate that this non-pneumophila spp. may not be as adept as L. pneumophila for intracellular uptake or replication within protozoa.

\subsection{The Intracellular Life Cycle of L. bozemanae Is Similar to That of L. pneumophila}

The intracellular lifestyle of L. pneumophila in protozoa is well established; subversion of the digestive pathway and formation of the replicative vacuole is dependent on the Dot/Icm pathway [40]. However, very little is known about the intracellular lifestyle of non-pneumophila species, such as L. bozemanae.

To gain further insight into Legionella uptake, growth rates, and fluorescence over time, we visualized Legionella infection in A. castellanii using confocal microscopy. At $6 \mathrm{~h}$ post-infection, bacteria can be seen within the host (Figure S1); however, A. castellanii infected with the L. pneumophila spp. tended to have more than 1 bacterium present per cell. At $12 \mathrm{~h}$ post-infection, the bacteria can be seen clustered together and dividing inside the cell, which is a process that appeared to be similar for both species (Figure 4). 


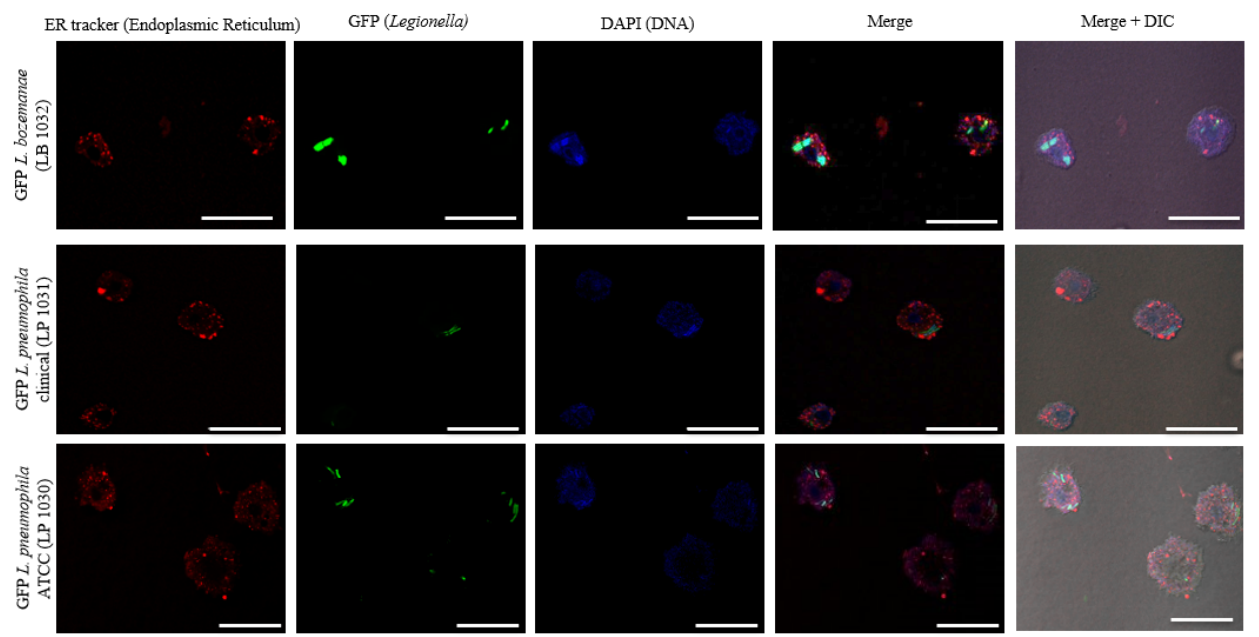

Figure 4. Confocal micrographs of $A$. castellanii infected with GFP-expressing Legionella strains. Image panels represent $A$. castellanii protozoa infected with GFP-expressing L. bozemanae, L. pneumophila, and L. pneumophila ATCC 33,152 strains $12 \mathrm{~h}$ post-infection visualized with a Zeiss LSM 700 confocal microscope. Individual fluorescence channels are displayed. The panel on the far right represents the merged fluorescence channels (green, GFP bacteria; red, endoplasmic reticulum; blue, DNA; and DIC). Scale bar $=10 \mu \mathrm{M}$. ATCC, American Type Culture Collection; DIC, Differential interference contrast; GFP, Green fluorescent protein.

\section{Discussion}

To date, research regarding Legionella has rarely focused on species outside of L. pneumophila SG1. Here, we report, for the first time, the generation of a GFP-expressing $L$. bozemanae strain derived from a clinical isolate obtained from our local hospital. Transformation did not appear to affect the growth kinetic profiles of the GFP-expressing Legionella strains compared to their parental counterparts.

This work extends our knowledge of non-pneumophila species by comparative studies of the growth profiles and relative fluorescence of L. bozemanae to L. pneumophila. Since the Legionella genus encompasses a diverse group of organisms, it is important to expand research to include non-pneumophila spp., particularly those that can cause disease [41-43]. In our study, several similarities and differences between L. pneumophila and L. bozemanae were seen. In broth, although each of the strains grew well in BYE at $37^{\circ} \mathrm{C}$, the L. pneumophila strains featured a faster growth rate with higher optical densities than that observed for L. bozemanae. These results match those observed in earlier studies [14,15,44], which reported that the non-pneumophila spp. often exhibit slower and reduced growth rates compared to L. pneumophila when grown in vitro at $37^{\circ} \mathrm{C}$.

In the protozoan model, the GFP-expressing L. bozemanae and L. pneumophila clinical strains, as well as the fluorescent L. pneumophila ATCC 33,152 strain, were all able to infect and replicate within A. castellanii. As amoeba share many similarities to human phagocytic cells, it was not surprising that the L. bozemanae clinical isolate was able to infect and replicate in the amoeba model. Although the GFP-expressing L. bozemanae strain did not attain growth levels as high as the GFP-expressing L. pneumophila strains, a steady increase in bacterial load was seen throughout the experiment. These findings differ from those of Neumeister and co-workers [45], which reported that L. bozemanae was not able to multiply within A. castellanii and rather showed a decreasing bacterial count in amoeba following infection. A possible explanation for these differing results may be attributed to strain differences as variability regarding infection rates, replication efficiency, and pathology has been documented, even within a single species [42,45,46]. Moreover, the lack of standardization regarding experimental conditions for Legionella infections in the $A$. castellanii infection model may also be a contributing factor.

Next, confocal microscopy was used to visualize Legionella spp. intracellular growth inside A. castellanii over time. L. bozemanae bacteria appear to replicate and cluster together, 
which may be indicative of vacuole formation, suggesting that the intracellular lifestyle of L. bozemanae may be similar to that of L. pneumophila. However, these findings need to be further defined through the use of detailed microscopy studies of L. bozemanae strains that are dysfunctional for genetic components known to be associated with pathogenesis as characterized by L. pneumophila.

Comparing the fluorescent intensities between the Legionella strains showed that the L. pneumophila strains, as comparative controls, featured stronger fluorescence than L. bozemanae even when normalizing fluorescence by optical density. Heterogeneity in fluorescence signals can occur due to several factors including differences in the copy numbers of the plasmids inside the cells, differences in plasmid protein expression, or poor GFP folding $[47,48]$. Nevertheless, the matched GFP expression profiles between the Legionella strains in this study with that previously reported by Hiltz and colleagues [49] indicate the recognition of a promoter region sourced from L. pneumophila by transcription and translation machinery in L. bozemanae, thereby allowing GFP expression. Despite the lower levels of GFP expression in L. bozemanae, the bacterial cells were bright enough to be seen clearly during intracellular infections of $A$. castellanii, and they can be used for further investigations of host-pathogen interactions. Further work is required to refine this approach by optimizing GFP expression through further genetic modifications of the plasmid as well as conducting whole genome sequencing to check for presence of any naturally occurring plasmids.

Taken together, this study shows the feasibility of using plasmid-borne GFP expression as a tool to investigate the intracellular lifestyle of lesser-known non-pneumophila Legionella species reported to cause Legionnaires' disease. Aside from the well-characterized L. pneumophila, and to a lesser extent, L. longbeachae, very little is known of the mechanisms employed by non-pneumophila Legionella species in causing disease in humans. The use of plasmid-borne GFP is advantageous over that of immunofluorescence, as the latter requires cell fixation as well as specific primary antibodies that may not be available for non-pneumophila Legionella species, whereas the former allows real-time monitoring of bacterial growth dynamics in vitro and in vivo. While antibiotic supplementation for plasmid maintenance and metabolic burden of GFP expression may be negative aspects of the use of GFP, these requirements do not seem to significantly affect the growth of L. bozemanae in vitro and in vivo, as observed in this study. Thus, the use of GFP as a fluorescent marker is beneficial to future studies on non-pneumophila Legionella species.

Supplementary Materials: The following are available online at https:/ / www.mdpi.com/2076-2 $607 / 9 / 2 / 379 /$ s1. Figure S1. Confocal micrographs of A. castellanii infected with GFP-expressing Legionella strains. Image panels represent $A$. castellanii infected with GFP-expressing L. bozemanae (LB 1032), L. pneumophila clinical (LP 1031), and L. pneumophila ATCC 33152 (LP 1030) strains $6 \mathrm{~h}$ post-infection visualized with an LSM 700 confocal microscope. Individual fluorescence channels are displayed. The panel on the far right represents the merged fluorescence channels (green, GFP bacteria; red, endoplasmic reticulum; blue, DNA; and DIC). Scale bar $=2 \mu \mathrm{M}$. ATCC, American Type Culture Collection; DIC, differential interference contrast; GFP, green fluorescent protein.

Author Contributions: B.M.H.: Conceptualization, investigation, methodology, data curation, analysis, writing-original draft preparation. C.I.G.: Methodology, writing-reviewing, and editing. T.M.: Methodology, writing-reviewing, and editing. Y.K.: supervision, writing-reviewing and editing, funding acquisition. A.K.C.B.: Conceptualization, project administration, resources, supervision, writing - reviewing and editing, funding acquisition. All authors have read and agreed to the published version of the manuscript.

Funding: This research was funded by NSERC, grant number RGPIN-2019-05490 which was awarded to A.K.C.B.

Institutional Review Board Statement: Not applicable.

Informed Consent Statement: Not applicable.

Data Availability Statement: Data is contained within the article or supplementary material. 
Conflicts of Interest: The authors declare no conflict of interest. The funders had no role in the design of the study; in the collection, analyses, or interpretation of data; in the writing of the manuscript, or in the decision to publish the results.

\section{References}

1. $\quad$ Benedict, K.M.; Reses, H.; Vigar, M.; Roth, D.M.; Roberts, V.A.; Mattioli, M.; Cooley, L.A.; Hilborn, E.D.; Wade, T.J.; Fullerton, K.E.; et al. Surveillance for waterborne disease outbreaks associated with drinking water- United States, 2013-2014. Morb. Mortal. Wkly. Rep. 2017, 66, 1216-1221. [CrossRef] [PubMed]

2. NNDSS Annual Report Wroking Group. Australia's Notifiable Disease Status, 2014: Annual Report of the National Notifiable Diseases Surveillance. System Commun. Dis. Intell. 2016, 40, 1.

3. European Centre for Disease Prevention and Control (ECDC) Surveillance Atlas-Legionnaires Disease. Available online: https:/ / www.ecdc.europa.eu/en/legionnaires-disease/surveillance/atlas (accessed on 25 January 2021).

4. Public Health Agency of Canada Legionellosis Reported Cases from 1986 to 2018 in Canada. Available online: https:/ / diseases. canada.ca/notifiable/charts?c=yl (accessed on 2 February 2021).

5. European Centre for Disease Prevention and Contol. Annual Epidemiological Report for 2017 Legionnaires' Disease; European Centre for Disease Prevention and Contol: Stockholm, Sweden, 2019.

6. Centers for Disease Control and Prevention. Legionellosis-United States, 2000-2009; Centers for Disease Control and Prevention: Atlanta, GA, USA, 2011; Volume 60.

7. Collier, S.A.; Deng, L.; Adam, E.A.; Benedict, K.M.; Beshearse, E.M.; Blackstock, A.J.; Bruce, B.B.; Derado, G.; Edens, C.; Fullerton, K.E.; et al. Estimate of burden and direct healthcare cost of infectious waterborne disease in the United States. Emerg. Infect. Dis. 2021, 27, 140-149. [CrossRef]

8. World Health Organization/Pan American Health Organization. Legionella and the Prevention of Legionellosis; World Health Organization: Geneva, Switzerland, 2007.

9. Cosson, P.; Soldati, T. Eat, kill or die: When amoeba meets bacteria. Curr. Opin. Microbiol. 2008, 11, 271-276. [CrossRef]

10. Moffat, J.F.; Tompkins, L.S. A quantitative model of intracellular growth of Legionella pneumophila in Acanthamoeba castellanii. Infect. Immun. 1992, 60, 296-301. [CrossRef] [PubMed]

11. Gast, R.J.; Moran, D.M.; Dennett, M.R.; Wurtsbaugh, W.A.; Amaral-Zettler, L.A. Amoebae and Legionella pneumophila in saline environments. J. Water Health 2011, 9, 37-52. [CrossRef] [PubMed]

12. www.bacterio.net LPSN. Available online: http:/ / www.bacterio.net/legionella.html (accessed on 5 July 2018).

13. Doleans, A.; Aurell, H.; Reyrolle, M.; Lina, G.; Freney, J.; Vandenesch, F.; Etienne, J.; Jarraud, S. Clinical and Environmental Distributions of Legionella Strains in France Are Different. J. Clin. Microbiol. 2004, 42, 458-460. [CrossRef]

14. Mercante, J.W.; Winchell, J.M. Current and emerging Legionella diagnostics for laboratory and outbreak investigations. Clin. Microbiol. Rev. 2015, 28, 95-133. [CrossRef]

15. Jarraud, S.; Descours, G.; Ginevra, C.; Lina, G.; Etienne, J. Identification of Legionella in Clinical Samples. In Legionella: Methods and Protocols; Buchrieser, C., Hilbi, H., Eds.; Springer: New York, NY, USA, 2013; Volume 945, pp. 27-56. ISBN 9781627032384.

16. Ng, V.; Tang, P.; Jamieson, F.; Guyard, C.; Low, D.E.; Fisman, D.N. Laboratory-based evaluation of legionellosis epidemiology in Ontario, Canada, 1978 to 2006. BMC Infect. Dis. 2009, 9, 1-10. [CrossRef]

17. Phin, N.; Parry-Ford, F.; Harrison, T.; Stagg, H.R.; Zhang, N.; Kumar, K.; Lortholary, O.; Zumla, A.; Abubakar, I. Epidemiology and clinical management of Legionnaires' disease. Lancet Infect. Dis. 2014, 14, 1011-1021. [CrossRef]

18. Head, B.M.; Trajtman, A.; Bernard, K.; Burdz, T.; Vélez, L.; Herrera, M.; Vanessa, Z.; Keynan, Y. Legionella co-infection in HIV-associated pneumonia. Diagn. Microbiol. Infect. Dis. 2019. [CrossRef] [PubMed]

19. Mancini, G.; Erario, L.; Gianfreda, R.; Oliva, A.; Massetti, A.P.; Mastroianni, C.M.; Vullo, V. Tuberculosis and Legionella pneumophila pneumonia in a patient receiving anti-tumour necrosis factor- a (anti-TNF- a) treatment. Eur. Soc. Clin. Microbiol. Infect. Dis. 2007, 13, 1036-1037. [CrossRef] [PubMed]

20. Jacobson, K.L.; Miceli, M.H.; Tarrand, J.J.; Kontoyiannis, D.P. Legionella pneumonia in cancer patients. Medicine (Baltimore) 2008, 87, 152-159. [CrossRef]

21. Just, S.A.; Knudsen, J.B.; Uldum, S.A.; Holt, H.M. Detection of Legionella bozemanae, a new cause of septic arthritis, by PCR followed by specific culture. J. Clin. Microbiol. 2012, 50, 4180-4182. [CrossRef] [PubMed]

22. Neiderud, C.-J.; Lagerqvist Vidh, A.; Salaneck, E. Soft tissue infection caused by Legionella bozemanii in a patient with ongoing immunosuppressive treatment. Infect. Ecol. Epidemiol. 2013, 3, 20739. [CrossRef]

23. Becton Dickinson and Company BCYE Agar. Quality Control Procedures; Becton Dickinson and Company: East Rutherford, NJ, USA, 2016; pp. 1-5.

24. Gogoi, S.K.; Gopinath, P.; Paul, A.; Ramesh, A.; Ghosh, S.S.; Chattopadhyay, A. Green fluorescent protein-expressing Escherichia coli as a model system for investigating the antimicrobial activities of silver nanoparticles. Langmuir 2006, 22, 9322-9328. [CrossRef]

25. Stretton, S.; Techkarnjanaruk, S.; Mclennan, A.M.; Goodman, A.E. Use of green fluorescent protein to tag and investigate gene expression in marine bacteria. Appl. Environ. Microbiol. 1998, 64, 2554-2559. [CrossRef] 
26. Morash, M.G.; Brassinga, A.K.C.; Warthan, M.; Gourabathini, P.; Garduño, R.A.; Goodman, S.D.; Hoffman, P.S. Reciprocal expression of integration host factor and HU in the developmental cycle and infectivity of Legionella pneumophila. Appl. Environ. Microbiol. 2009, 75, 1826-1837. [CrossRef]

27. Hoffmann, C.; Harrison, C.F.; Hilbi, H. The natural alternative: Protozoa as cellular models for Legionella infection. Cell. Microbiol. 2014, 16, 15-26. [CrossRef]

28. Barbier, M.; Damron, F.H. Rainbow vectors for broad-range bacterial fluorescence labeling. PLoS ONE 2016, 11, 1-16. [CrossRef]

29. Tombolini, R.; Unge, A.; Davey, M.E.; De Bruijn, F.J.; Jansson, J.K. Flow cytometric and microscopic analysis of GFP-tagged Pseudomonas fluorescens bacteria. FEMS Microbiol. Ecol. 1997, 22, 17-28. [CrossRef]

30. Aye, T.; Wachsmuth, K.; Feeley, J.C.; Gibson, R.J.; Johnson, S.R. Plasmid profiles of Legionella species. Curr. Microbiol. 1981, 6, 389-394. [CrossRef]

31. Kain, S.R. Methods and protocols. Methods Biochem. Anal. 2005, 47, 407-421. [CrossRef]

32. Feeley, J.C.; Gibson, R.J.; Gorman, G.W.; Langford, N.C.; Rasheed, J.K.; Mackel, D.C.; Baine, W.B. Charcoal-yeast extract agar: Primary isolation medium for Legionella pneumophila. J. Clin. Microbiol. 1979, 10, 437-441. [CrossRef] [PubMed]

33. Dennis, J.J.; Zylstra, G.J. Plasposons: Modular self-cloning minitransposon derivatives for rapid genetic analysis of gram-negative bacterial genomes. Appl. Environ. Microbiol. 1998, 64, 2710-2715. [CrossRef]

34. Bachman, M.A.; Swanson, M.S. Genetic evidence that Legionella pneumophila RpoS modulates expression of the transmission phenotype in both the exponential phase and the stationary phase. Infect. Immun. 2004, 72, 2468-2476. [CrossRef]

35. Tanner, J.R.; Li, L.; Faucher, S.P.; Brassinga, A.K.C. The CpxRA two-component system contributes to Legionella pneumophila virulence. Mol. Microbiol. 2016, 100, 1017-1038. [CrossRef]

36. Berger, K.H.; Isberg, R.R. Two distinct defects in intracellular growth complemented by a single genetic locus in Legionella pneumophila. Mol. Microbiol. 1993, 7, 7-19. [CrossRef] [PubMed]

37. Pitre, C.A.J.; Tanner, J.R.; Patel, P.; Brassinga, A.K.C. Regulatory control of temporally expressed integration host factor (IHF) in Legionella pneumophila. Microbiol. (U. K.) 2013, 159, 475-492. [CrossRef] [PubMed]

38. Dietersdorfer, E.; Cervero-Aragó, S.; Sommer, R.; Kirschner, A.K.; Walochnik, J. Optimized methods for Legionella pneumophila release from its Acanthamoeba hosts. BMC Microbiol. 2016, 16, 1-10. [CrossRef]

39. Schneider, C.A.; Rasband, W.S.; Eliceiri, K.W. NIH Image to ImageJ: 25 years of image analysis. Nat. Methods 2012, 9, 671-675. [CrossRef] [PubMed]

40. Miyake, M. Intracellular survival and replication of legionella pneumophila within host cells. Yakugaku zasshi J. Pharm. Soc. Japan 2008, 128, 1763-1770. [CrossRef]

41. Joshi, A.D.; Swanson, M.S. Comparative analysis of Legionella pneumophila and Legionella micdadei virulence traits. Infect. Immun. 1999, 67, 4134-4142. [CrossRef]

42. Terry Alli, O.A.; Zink, S.; von Lackum, N.K.; Abu-kwaik, Y. Comparative assessment of virulence traits in Legionella spp. Microbiology 2003, 149, 631-641. [CrossRef]

43. Qin, T.; Zhou, H.; Ren, H.; Liu, W. Distribution of secretion systems in the genus Legionella and its correlation with pathogenicity. Front. Microbiol. 2017, 8, 1-12. [CrossRef]

44. Wang, C.; Saito, M.; Tanaka, T.; Amako, K.; Yoshida, S. ichi Comparative analysis of virulence traits between a Legionella feeleii strain implicated in Pontiac fever and a strain that caused Legionnaires' disease. Microb. Pathog. 2015, 89, 79-86. [CrossRef] [PubMed]

45. Neumeister, B.; Schöniger, S.; Faigle, M.; Eichner, M.; Dietz, K. Multiplication of different Legionella species in Mono Mac 6 cells and in Acanthamoeba castellanii. Appl. Environ. Microbiol. 1997, 63, 1219-1224. [CrossRef] [PubMed]

46. Newton, H.J.; Ang, D.K.Y.; Van Driel, I.R.; Hartland, E.L. Molecular pathogenesis of infections caused by Legionella pneumophila. Clin. Microbiol. Rev. 2010, 23, 274-298. [CrossRef] [PubMed]

47. Kjos, M.; Aprianto, R.; Fernandes, V.E.; Andrew, P.W.; Van Strijp, J.A.G.; Nijland, R.; Veening, J.W. Bright fluorescent Streptococcus pneumoniae for live-cell imaging of host-pathogen interactions. J. Bacteriol. 2015, 197, 807-818. [CrossRef] [PubMed]

48. Jørgensen, M.G.; van Raaphorst, R.; Veening, J.W. Noise and Stochasticity in Gene Expression. A Pathogenic Fate Determinant. Methods Microbiol. 2013, 40, 157-175. [CrossRef]

49. Hiltz, M.F.; Sisson, G.R.; Brassinga, A.K.C.; Garduno, E.; Garduno, R.A.; Hoffman, P.S. Expression of magA in Legionella pneumophila Philadelphia-1 Is Developmentally Regulated and a Marker of Formation of Mature Intracellular Forms. J. Bacteriol. 2004, 186, 3038-3045. [CrossRef] [PubMed] 